\title{
Study the Z-plane strip capacitance
}

\author{
Harshil Parikh \\ Office of Science, Science Undergraduate Laboratory Internship \\ University of Illinois at Urbana-Champaign \\ Stanford Linear Accelerator Center \\ Menlo Park, CA
}

August $4^{\text {th }}, 2005$

Prepared in partial fulfillment of the requirements of the Office of Science, Department of Energy's Science Undergraduate Laboratory Internship under the direction of Dr. Sanjay Swain in the High-Energy department at Stanford Linear Accelerator Center.

Participant:

Research Advisor: 


\section{Table of Contents}

$\begin{array}{ll}\text { Abstract } & 3-4 \\ \text { Introduction } & 4-6 \\ \text { Materials and Methods } & 6-11 \\ \text { Results } & 11-14 \\ \text { Discussion and Conclusions } & 15-16 \\ \text { Acknowledgements } & 16 \\ \text { References } & 17\end{array}$




\begin{abstract}
Study of the Z-plane strip capacitance. Harshil Parikh (University of Illinois at Urbana-Champaign, Urbana, IL 61801) Dr. Sanjay Swain (Stanford Linear Accelerator Center, Menlo Park CA 94305)

The BaBaR detector at the Stanford Linear Accelerator Center is currently undergoing an upgrade to improve its muon and neutral hadron detection system. The Resistive Plate Chambers (RPCs) that had been used till now have deteriorated in performance over the past few years and are being replaced by Limited Streamer Tube (LSTs). Each layer of the system consists of a set of up to 10 streamer tube modules which provide one coordinate ( $\phi$ coordinate) and a single "Z-plane" which provides the Z coordinate of the hit. The large area Zplanes (up to $12 \mathrm{~m}^{2}$ ) are $1 \mathrm{~mm}$ thick and contain 96 copper strips that detect the induced charge from avalanches created in the streamer tube wires. All the Zplanes needed for the upgrade have already been constructed, but only a third of the planes were installed last summer. After installing the 24 Z-planes last year, it was learned that $0.7 \%$ of the strips were dead when put inside the detector. This was mainly due to the delicate solder joint between the read-out cable and the strip, and since it is difficult to access or replace the Z-planes inside the detector, it is very important to perform various tests to make sure that the Z-
\end{abstract}


planes will be efficient and effective in the long term. We measure the capacitance between the copper strips and the ground plane, and compare it to the theoretical value that we expect. Instead of measuring the capacitance channel by channel, which would be a very tedious job, we developed a more effective method of measuring the capacitance. Since all the Z-planes were built at SLAC, we also built a smaller $46 \mathrm{~cm}$ by $30 \mathrm{~cm}$ Z-plane with 12 strips just to see how they were constructed and to gain a better understanding about the solder joints.

\section{Introduction}

The BaBar detector at Stanford Linear Accelerator Center (SLAC) is a device that studies $e^{+} e^{-}$collisions at the $\Upsilon(4 s)$. The detector group proposed an upgrade in May 2003 which was intended to improve muon and neutral hadron detection in the Instrumental Flux Return (IFR) barrel region of the BaBar detector. Muon detection is vital for analyses such as semi-/leptonic decays and lepton tagging for CP violation. Initially, Resistive Plate Chambers (RPCs) were used, but several persistent problems in the RPCs resulted in continuous drop in the efficiency of the muon identification. To improve the efficiency, the proposed upgrade introduced a completely new technology of Limited Streamer Tubes (LSTs). There are two components of LSTs: the streamer tube chamber array and an associated Z-plane made up of copper strips and copper planes. A gas flows through the streamer tubes, and when a $\mu$ passes through the tube, it 
ionizes the gas which produces electron/ion pairs. The electric field causes the pairs to accelerate towards the anode which causes the electrons to gain energy. This gain causes further ionization, and high voltage causes large amounts of charge multiplication (Townsend effect) that results in an avalanche. The secondary electron/ion pairs create an electric field comparable to the applied field. The avalanche gets saturated, but there is an electric field between the avalanche tip and the anode wire. New avalanches form from photoionized electrons, and the streamer propagates to the anode wire producing a signal. A current is also induced in the Z-plane below the tubes, and this current is detected through the cable soldered with the Cu strip.

During the summer of 2004, streamer tubes and Z-planes were installed in 2 of the 6 sections of the IFR barrel region. Several important lessons were learned while installing the 24 Z-planes in the detector. Firstly, handling and moving the Z-planes sometimes broke the solder joint, and these broken solder joints were only found after there put into the detector. Secondly, a device was needed to quickly and efficiently check whether the solder joints are broken before we put them into the the detector. The Z-planes are made in a novel but complicated method using mylar sheets, copper strips, ribbon cables, and copper sheets. The copper strips have been soldered to the ribbon cables, but from previous experience, they are known to easily break and make that part of the Zplane essentially useless. In order to solve this problem, we measure the 
capacitance of the Z-planes with respect to the ground plane, and we use the results of the capacitance to determine the status of the solder joints.

\section{Materials and Methods}

Since the Z-plane consists of a $\mathrm{Cu}$ ground plane below the $96 \mathrm{Cu}$ strips, one can theoretically approximate the capacitance between a strip and the plane, and measure it experimentally. An approximate formula to calculate the

capacitance between the strip and the plane is given by: $C=\frac{2 \varepsilon_{o} A_{1} A_{2}}{\left(A_{1}+A_{2}\right) d}$ where $A_{1}$ is the area of the strip, $A_{2}$ is the area of the ground plane and $d$ is the distance between them. If one plugs in an approximate value for the three variables, one gets an approximate capacitance value $C \approx 4.55 n F$. Since the ribbon cable that carries the signal is soldered to the Cu strip, the capacitance between the ribbon cable and the ground plane should approximately be equal to the capacitance between the Cu strip and the ground plane. Therefore, one can conclude that if one measures the capacitance between the ribbon cable and the ground plane, one should measure a value close to $C \approx 4.55 \mathrm{nF}$. For any given strip in the Z-plane, if the solder joint is intact and the strip is well connected to the cable, one should measure a capacitance value close to the calculated approximate value. On the other hand, if one measures a capacitance value that is much less than the theoretical value, then one can conclude that the solder joint is loose and it must be fixed before the Z-planes are installed inside the 
detector. Since there were 48 Z-planes (4 Layer 18 Z-planes) that need to be installed, and each Z-plane has 96 strips (except Layer 18), thats a total of 4568 strips, or in other words, 4568 measurements. We realized that with a little ingenuity, we can take these measurements in a much more effective manner than manually measuring channel-by-channel using a capacitance meter. Moreover, if we can measure the capacitance of one Z-plane in a time efficient manner, we can check for bad solder joints very quickly and that would help us tremendously during installation. If we build an electrical box that takes in the signal from the ribbon cable, and use a switch to swap between the 16 channels, we can take these 4568 measurements in an effective manner. In order to build the box, several materials were needed: 2 12-position single pole rotary switchs, a 16 channel signal cable, a transition board, an output BNC connector, a capacitance meter, and a small box to mount the switches and the connector.

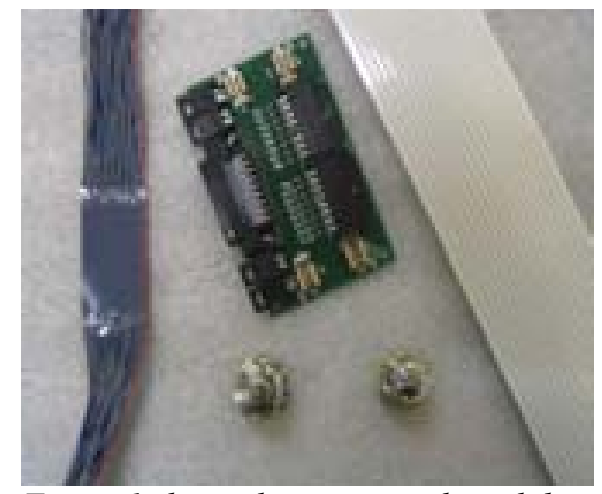

Figure 1 shows the transition board that testing, it is determined that the white color takes in the white ribbon cable from the $Z$-plane, a 16 channel blue \& white signal wires are the signal wires, and now, we needed
cable and 2 12-position single pole rotary switches
Figure 1 shows most of the materials that were used to build the box. We first strip the insulation off the blue and white signal cable and determine which color wires carry the signal and which color wires act as ground. After some to figure out how to solder to solder on 16 
signal wires on the 212 -position rotary switches. Since the switch has 12 input and one output positions, we decided to do the following: solder 11 signal cables (first white cable onto position 1 , second white cable onto position 2 , etc.), onto positions $1-11$ on the switch (switch A), then use another wire to connect the out of the $2^{\text {nd }}$ switch (switch B) onto the $12^{\text {th }}$ position of the first switch. Then, if we connect the remaining 5 signal cables onto position $1-5$ on the second switch, we have completely connected the 16 channel signal cable to the 2 rotary switches. We now collected all the ground wires together (blue wires), and soldered them onto the box using a solder log. The solder log is then screwed onto the box, hence acting as a ground. We then took another wire and connected the output of the first switch (the one with 11 signal wires) to a BNC output connector. We then use a BNC wire cable to connect from the BNC connector to the capacitance meter, which than reads out the value of the capacitance. One final step was needed to connect the ground plane to the box: we took another wire and soldered it to a solder log, and connected the other end of the wire to an alligator clip. Now, we can clip the ground plane to the box using the wire with the alligator clip, and our box is complete.

Figure 2 shows the final picture of the box, with all its components. After the box has been completeld, we needed to figure out a convention on how to rotate the switch to swap between channels $1-16$. Since 11 signal wires are connected to one switch, channels $1-11$ can be accessed by rotating switch $A$ 


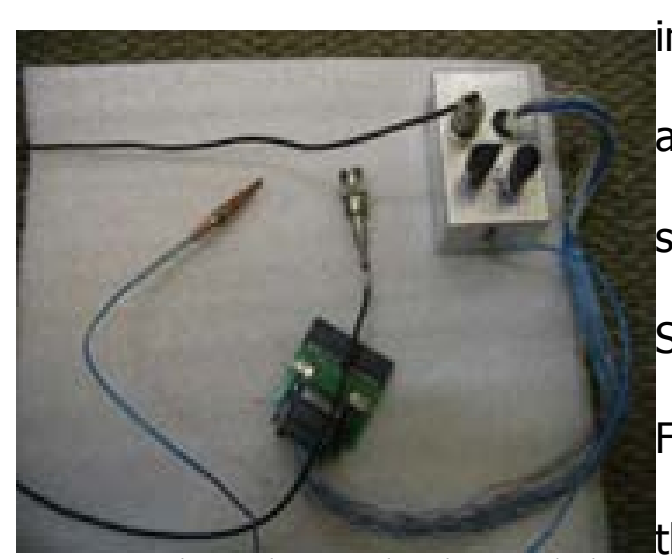

in the counterclockwise direction. In order to access channels $12-16$, one needs to place switch $A$ on position 12, and then rotate Switch B in the counterclockwise direction. Finally then, we now had a capacitance box that we could use to measure the capacitance Figrure 2 shows the complete box, with the 16 channel signal cable connected to the transition board, the blue wire with the alligator clip that connects to the ground plane, and the black wire which connects from an out BNC connector to the capacitance meter between the output ribbon cables and the $\mathrm{Cu}$ ground plane. In order to take a measurement, we insert the 2 ribbon cables from the Z-plane into the transition board, attach the BNC cable to a capacitance meter, and rotate the switches to get the measurements.

A second major part of my project involved actually making a small Zplane. This small Z-plane had 16 strips (8 on each side), and it was made using the same materials as the actual Z-planes that were made last year. The motivations for making the Z-plane are multi-fold: to grasp a better understanding about the structure of the Z-plane, to learn more about the solder joints and why they are so delicate and break easily, and to gain new knowledge in a novel technique of vacuum thermal lamination. Figure 3 shows the precise makeup of the Z-plane, and we followed this structure while making our smaller Z-plane. We first cut out $3 \mathrm{mil}, 5 \mathrm{mil}$, and 10 mil mylar film to the size of the Zplane $(60 \mathrm{~cm} \times 24 \mathrm{~cm})$, and a Cu foil just a bit smaller than the size of the Mylar 
film. We first placed the 3 Mil Mylar adhesive side up, then placed a $\mathrm{Cu}$ Foil on top of the mylar. We then added a 10 Mil mylar adhesive side face down, another 10 Mil mylar adhesive side face down with holes, and 2 ribbon cables with solder on

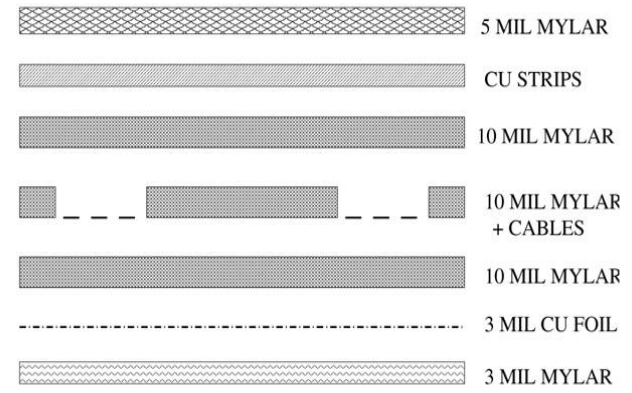

Figure 3: Detailed make up of the Z-plane them at 8 different wires at 8 spots.

Therefore, each cable had 8 solder spots on 8 different wires (1 solder spot per wire), and these solder spots matched the holes in the 10 mil mylar. Then, we added Cu strips

on top of the 10 Mil mylar film with holes, so that we could feel the solder spots from the cable to the strips. We then carefully cut out the $\mathrm{Cu}$ strip just enough so that the solder spot passes through the strip, and then we melted the solder spot onto the $\mathrm{Cu}$ strip. The ribbon cable was now soldered onto the $\mathrm{Cu}$ strip, an we added another 5 mil mylar adhesive side face down on top of the Cu strip to protect the strip (Figure 4). We now needed to take all the air out from the Zplane, so we used this new process of thermal vacuum lamination to suck all the air out from the plane. 


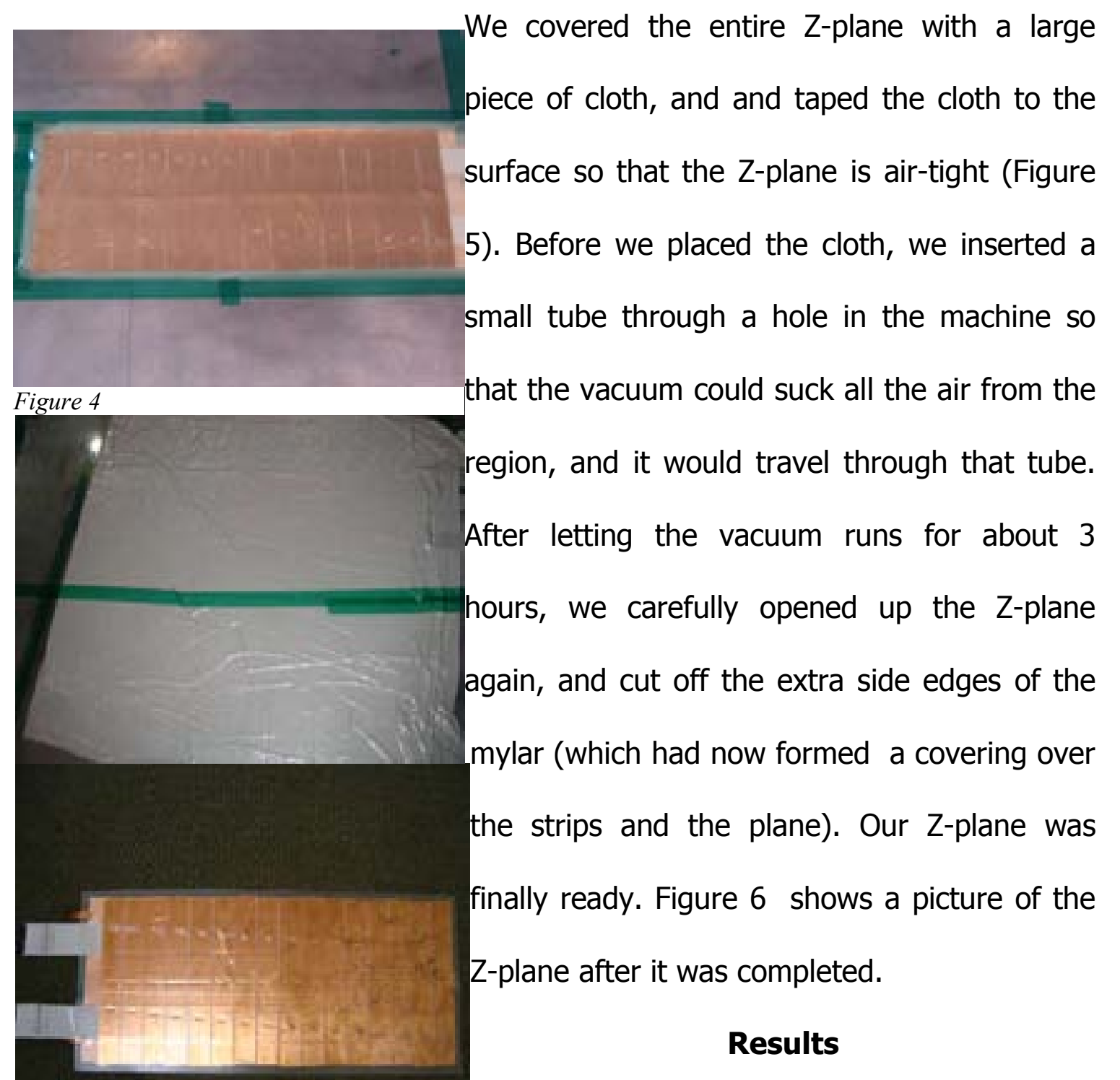

Since the capacitance of the Z-planes had never been studied before, we thought it was Figure 4, 5, 6: Z-plane before cloth was put on; Z-plane covered in cloth and taped to make it air tight; Z-plane after the thermal vacuum lamination process

a good idea to take some measurements manually first, instead of using the box right away. Firstly, by taking measurement manually first, we were able to get a good idea of the capacitance measurements as we measure from channel $1-96$. We 
took hand-by-hand measurements of 12 Z-planes, and the graph below shows the capacitance (in $\mathrm{nF}$ ) as a function of channel number for 4 of the $12 \mathrm{Z}$-planes. This graph proves that our theoretical value of $C \approx 4.55 n F$ is a good approximation for the capacitance, since most of the capacitance values for these 384 strips (4 planes $\times 96$ strips) lies close it.

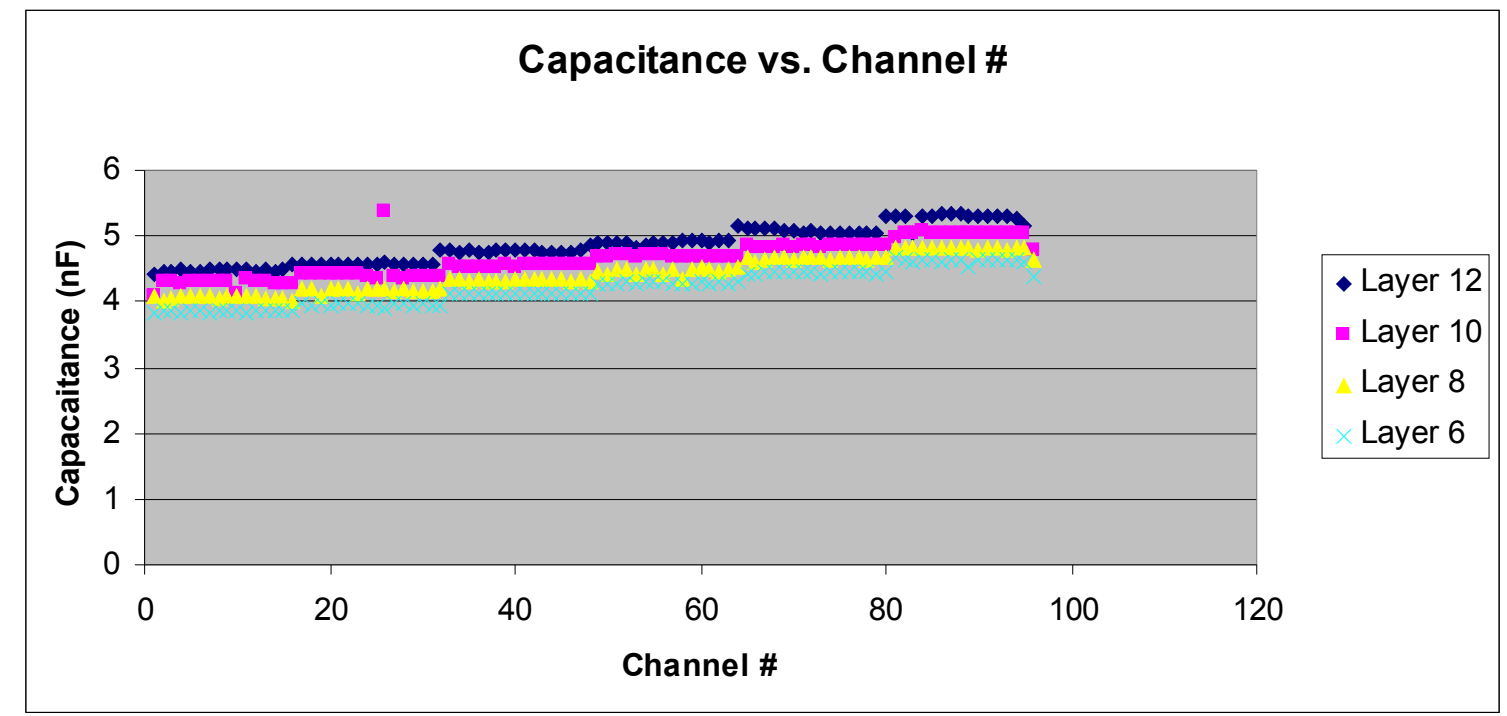

Picture1 1Capacitance vs. Channel \#

This graph also proves our suspicions that the cable length does affect the capacitance. Since the length of the cable is equal for 16 strips, and then increases for the $17^{\text {th }}$ one, it makes sense that the capacitance stays constant for the first 16 channels and then increases for the $17^{\text {th }}$ one.

Since we took capacitance measurements for 12 Z-planes manually, we used this data to check whether our box is working or not. We took capacitance measurments of the same 12 Z-planes using the box, and compared our 
measurements to those from the manual data. The graph below compares the capacitance measurements for one layer.

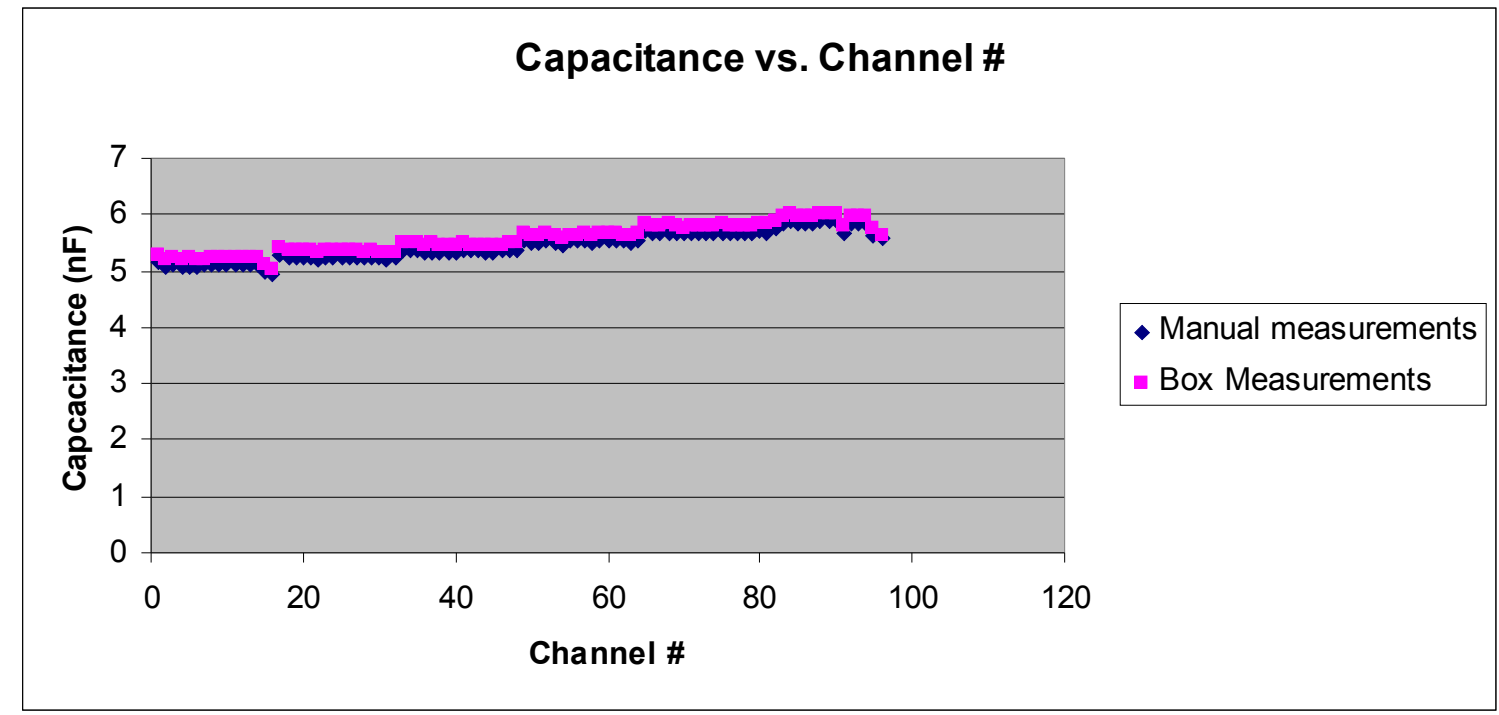

Picture1 2Capacitance vs. Channel \#

The graph above shows that our box is working well. The manual data and the box data follow the same pattern for one of the layers measured, except for an additional constant in all measurements in the box data. This graph proves to us that we can take measurements of the remaining 36 planes using the capacitance box. After taking measurements of the remaining 36 planes, I was able to form a loose relationship between capacitance value and the status of the solder joint. The table below describes this relationship, and shows the status of all the solder joints from the 48 Z-planes.

\begin{tabular}{|c|l|c|}
\hline Capacitance $(\boldsymbol{n} \boldsymbol{F})$ & \multicolumn{1}{|c|}{ Status } & \multicolumn{2}{c|}{ Total \# } \\
\hline $3.0-6.5$ & Good joint & \\
\hline
\end{tabular}




\begin{tabular}{|l|l|r|}
\hline \multicolumn{1}{|c|}{ Capacitance $(\boldsymbol{n F})$} & \multicolumn{1}{|c|}{ Status } & \multicolumn{1}{c|}{ Total \# } \\
\hline$<3.0$ & Loose joint & 5 \\
\hline$>3.0$ & 2 shorted strips & 2 \\
\hline
\end{tabular}

Table 1Shows the total number of good and bad joints

The 5 joints that were loose were easy to find because their capacitance measurement was $C<2.0 \mathrm{nF}$. The graph below shows the capacitance measurements of one of the 4 layer 18 Z-planes. This Z-plane had 2 loose solder joints, as seen from this graph:

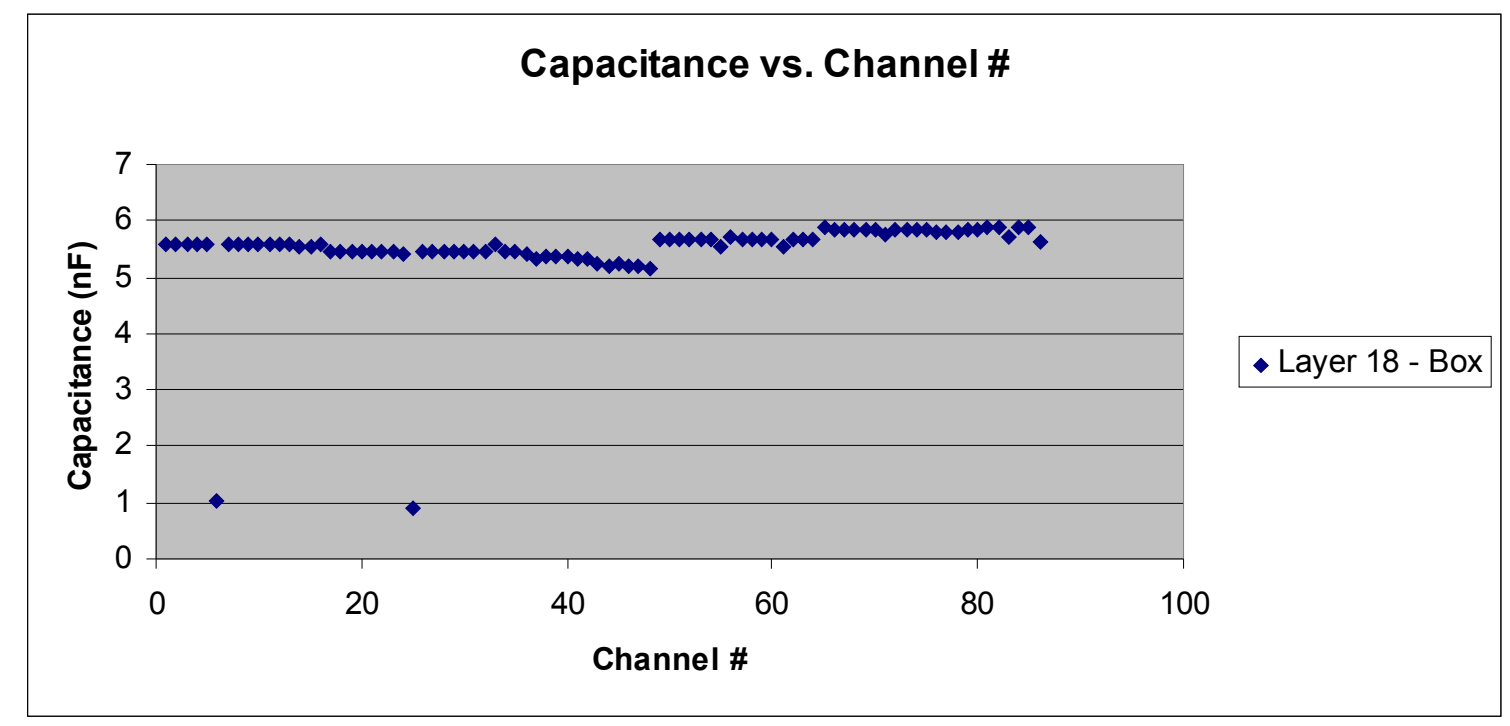

Picture1 3Capacitance vs. Channel \#

This graph clearly shows the 2 loose solder joints. These results were verified when we pressed these particular strips near the solder joint, the capacitance suddently increased, but again decreased when we stopped pressing near the solder joint. 


\section{Discussion and Conclusion}

Using the capacitance box was clearly a more efficient way to taking the capacitance measurements of these Z-planes. When we took the measurements manually, 12 Z-planes required 10 hours and two people to complete the job, while I was able to take capacitance measurements of 48 Z-planes in 10 hours without using anyone's help using the box. Using the box, I was able to finish my measurements almost $4 x$ faster. Before I could use the box, however, I had to make sure that the box was working correctly since there are many delicated solder joints attached to the switch and the BNC connector and the chances of mechanical errors are high. The agreement between the manual and box measurements allowed us to conclude that the box was working accurately. All the measurements from the box were a little higher than the manual meausements, and the source of this additive constant of $\quad C \approx 0.12 n F \quad$ is from the white signal cable wires that have been soldered to the switch. The cables have a small capacitance of their own, but since it is present in the measurements of every strip, we can ignore it. After measuring all the $48 \mathrm{Z}$ planes, our results show that a total of 7 were found as bad joints. 5 of the 7 bad joints were loose, and therefore, they must be resoldered before the next installation. The remaining 2 joints were shorted together, and these will be harder to fix. Somehow, these joints have been connected together and we need to find it, which will certainly not be easy. Nevertheless, this is not a bad result 
considering such a large number of Z-planes since we are still only $0.15 \%$ inefficient. While making the Z-planes, I realized how delicate the solder joint is and how easily it could break or disconnect from the strip. Since the solder joint is melted onto the Cu strip, if the melting does not cover the entire hole, the solder joint could potentially break very easily while moving and handling the Zplane. Since the installation for the remaining streamer tubes and Z-planes is not planned till next summer, continous testing of the Z-plane capacitance while they move around from one place to another will be necessary. Once these solder joints are fixed, regular testing will probably be helpful in order to gain a better understanding of how Z-planes withstand movement. When installation time does come next summer, the box will be of tremendous help to determine the status of a solder joint quickly before putting the Z-planes into the detector.

\section{Acknowledgements}

This research was conducted at the Stanford Linear Accelerator Center. I would like to thank the United States Department of Energy, Office of Science for giving me the opportunity to participate in such a learning and rewarding research internship. I would like to thank Dr. Sanjay Swain for guiding me in the right direction throughout my project, challenging my intellectual curiosity , and helping me in making my research experience a great learning experience. I would also like to thank Dr. Mark Convery, Dr. Charlie Young, and Dr. Wolfgang Menges for their continous support and help throughout my project. 


\section{References}

[1] BABAR Collaboration LST Team, “A Barrel IFR Instrumented with Limited Streamer Tubes," Stanford Linear Accelerator Center Experimental Program Advisory Committee, Stanford, CA, May 2003.

[2] S. Smith, "Limited Streamer Tubers for the IFR Barrel," Stanford Linear Accelerator Center Experimental Program Advisory Committee, Stanford, CA, Jun. 2003. 